\title{
Bi-amalgamations subject to the clean and nil-clean properties
}

\author{
Khalid Adarbeh*1 (D), Mohammad Adarbeh² (D) \\ ${ }^{1}$ Department of Mathematics, Faculty of Science, An-Najah National University, Nablus, Palestine \\ ${ }^{2}$ Department of Mathematics, Hebron University, Hebron, Palestine
}

\begin{abstract}
This paper investigates necessary and sufficient conditions for a bi-amalgamation to inherit the clean as well as the nil-clean properties. The new results recovers different settings of other constructions such as duplications and amalgamations. All results are used to build new and illustrative examples arising as bi-amalgamations.
\end{abstract}

Mathematics Subject Classification (2020). Primary 13C10, 13C11, 13E05, 13F05, 13H10; Secondary 16A30, 16A50, 16A52

Keywords. bi-amalgamation of a ring along ideals, (uniquely) clean rings, nil-clean rings, boolean rings

\section{Introduction}

All rings considered in this paper are commutative with unity. Let $f: A \rightarrow B$ and $g: A \rightarrow C$ be two commutative ring homomorphisms and let $J$ and $J^{\prime}$ be two ideals of $B$ and $C$, respectively, such that $f^{-1}(J)=g^{-1}\left(J^{\prime}\right)$. The bi-amalgamation of $A$ with $(B, C)$ along $\left(J, J^{\prime}\right)$ with respect to $(f, g)$ is the subring of $B \times C$ given by

$$
A \bowtie^{f, g}\left(J, J^{\prime}\right):=\left\{\left(f(a)+j, g(a)+j^{\prime}\right) \mid a \in A,\left(j, j^{\prime}\right) \in J \times J^{\prime}\right\} .
$$

This construction was introduced and studied by S. Kabbaj, K. Louartiti, and M. Tamekkante in [15]. It is a generalization of the amalgamated duplication (introduced and studied by DAnna and Fontana [10,11]) and the amalgamated algebras along an ideal (introduced and studied by M. DAnna, C. Finocchiaro and M. Fontana [7-9]). For more information about the bi-amalgamation, we refer the reader to $[3,15,16]$. Let $A$ and $B$ be rings, let $J$ be an ideal of $B$ and let $f: A \rightarrow B$ be a ring homomorphism. The amalgamation of $A$ with $B$ along $J$ with respect to $f$ is defined to be the subring of $A \times B$ :

$$
A \bowtie^{f} J:=\{(a, f(a)+j) \mid a \in A, j \in J\} .
$$

By [15, Example 2.1], $A \bowtie^{f} J=A \bowtie^{\iota, f}(I, J)$, where $I=f^{-1}(J)$ and $\iota=i d_{A}$. For more information about the amalgamation, we refer the reader to [4,6-11].

Recall that a ring $R$ is called (uniquely) clean if each element in $R$ can be written (uniquely) as the sum of a unit and an idempotent. The concept of clean rings was introduced by Nicholson [17]. Examples of clean rings (uniquely clean rings) include all

\footnotetext{
*Corresponding Author.

Email addresses: khalid.adarbeh@najah.edu (K. Adarbeh), adarbehm@hebron.edu (M. Adarbeh)

Received: 14.08.2021; Accepted: 15.09.2021
} 
commutative von Neumann regular rings (Boolean rings), any homomorphic image of a clean ring is again clean. A ring $R$ is called nil-clean if each of its elements can be written as the sum of an idempotent and a nilpotent. The notion of nil-clean ring was introduced by A. J. Diesl in [12], where he proved that the class of nil clean rings is strictly contained in the class of clean rings. Any Boolean ring is nil-clean and any homomorphic image of a nil-clean ring is again nil-clean. The following diagram of implications displays the relations among the mentioned concepts, where all the implications are irreversible in general.

\section{Boolean rings $\Rightarrow$ nil-clean rings $\Rightarrow$ clean rings}

In 2014, Mohamed Chhiti, Najib Mahdou, and Mohammed Tamekkante gave a characterization for the amalgamation of $A$ with $B$ along $J$ with respect to $f\left(A \bowtie^{f} J\right)$ to be clean. Namely; they proved that if $\frac{f(A)+J}{J}$ is uniquely clean, then $A \bowtie^{f} J$ is clean if and only if $A$ and $f(A)+J$ are clean rings. [5]. Then, in 2018, Chahrazade Bakkari and Mohamed Es-Said gave a characterization for $A \bowtie^{f} J$ to be nil-clean. Namely, $A \bowtie^{f} J$ is nil-clean if and only if $A$ and $f(A)+J$ are nil-clean rings. [2].

This paper establishes necessary and sufficient conditions for a bi-amalgamation $A \bowtie^{f, g}$ $\left(J, J^{\prime}\right)$ to inherit the clean property as well as the nil-clean property. All obtained results recover and compare to previous works carried on the amalgamations in $[2,5]$.

Throughout, $\operatorname{Nil}(A)$ denotes the nilradical of $A ; \operatorname{Id}(A)$ denotes the set of all idempotents of $A ; U(A)$ denotes the set of all units of $A$.

\section{Transfer results in the bi-amalgamation}

We begin with then following preliminary lemmas.

Lemma 2.1 ([15, Proposition 4.1 parts (2) and (3)]). Let $A \bowtie^{f, g}\left(J, J^{\prime}\right)$ be the biamalgamation of $A$ with $(B, C)$ along $\left(J, J^{\prime}\right)$ with respect to $(f, g)$ and let $I_{\circ}=f^{-1}(J)=$ $g^{-1}\left(J^{\prime}\right)$. Then

(1) $\frac{A \bowtie^{f, g}\left(J, J^{\prime}\right)}{0 \times J^{\prime}} \cong f(A)+J$ and $\frac{A \bowtie^{f, g}\left(J, J^{\prime}\right)}{J \times 0} \cong g(A)+J^{\prime}$.

(2) $\frac{A}{I_{\circ}} \cong \frac{A \bowtie^{f, g}\left(J, J^{\prime}\right)}{J \times J^{\prime}} \cong \frac{f(A)+J}{J} \cong \frac{g(A)+J^{\prime}}{J^{\prime}}$.

Lemma 2.2. Using the notation of Lemma 2.1,

If $a \in U(A), f(a)+j \in U(B)$, and $g(a)+j^{\prime} \in U(C)$, then $\left(f(a)+j, g(a)+j^{\prime}\right) \in$ $U\left(A \bowtie^{f, g}\left(J, J^{\prime}\right)\right)$.

Proof. Since $f(a)+j \in U(B)$, and $g(a)+j^{\prime} \in U(C)$, there are $b \in B$ and $c \in C$ such that $(f(a)+j) b=1$ and $\left(g(a)+j^{\prime}\right) c=1$. So

$$
\left(f(a)+j, g(a)+j^{\prime}\right)\left(f\left(a^{-1}\right)-b f\left(a^{-1}\right) j, g\left(a^{-1}\right)-c g\left(a^{-1}\right) j^{\prime}\right)=(1,1) .
$$

From now until the end of this article, in all the results we will be using the notation of Lemma 2.1. Assume that $A$ is clean ring and $\frac{A}{I_{\circ}}$ is uniquely clean ring (using the notation of Lemma 2.1). The following theorem establishes the necessary and sufficient conditions under which the bi-amalgamation $A \bowtie^{f, g}\left(J, J^{\prime}\right)$ will be clean.

Theorem 2.3. Consider $A \bowtie^{f, g}\left(J, J^{\prime}\right)$, where $A$ is clean.

(1) If $A \bowtie^{f, g}\left(J, J^{\prime}\right)$ is clean, then $f(A)+J$ and $g(A)+J^{\prime}$ are clean rings.

(2) Suppose that $\frac{A}{I_{\circ}}$ is uniquely clean, then $A \bowtie^{f, g}\left(J, J^{\prime}\right)$ is clean if and only if $f(A)+J$ and $g(A)+J^{\prime}$ are clean rings

Proof. (1) Since $f(A)+J$ and $g(A)+J^{\prime}$ are homomorphic image of $A \bowtie^{f, g}\left(J, J^{\prime}\right)$ (by Lemma 2.1) and $A \bowtie^{f, g}\left(J, J^{\prime}\right)$ is clean, then they are clean. 
(2) Let $a \in A$ and $\left(j, j^{\prime}\right) \in J \times J^{\prime}$. Then $a=u+e$, where $u \in U(A)$ and $e \in I(A)$ (since $A$ is clean). Since $f(A)+J$ and $g(A)+J^{\prime}$ are clean, then $f(a)+j=$ $f\left(x_{1}\right)+j_{1}+f\left(x_{2}\right)+j_{2}$ and $g(a)+j^{\prime}=g\left(y_{1}\right)+j_{1}^{\prime}+g\left(y_{2}\right)+j_{2}^{\prime}$, where $f\left(x_{1}\right)+j_{1}$ and $f\left(x_{2}\right)+j_{2}\left(g\left(y_{1}\right)+j_{1}^{\prime}\right.$ and $\left.g\left(y_{2}\right)+j_{2}^{\prime}\right)$ are respectively unit and idempotent elements of $f(A)+J\left(g(A)+J^{\prime}\right)$. Clearly, $\overline{f\left(x_{1}\right)}=\overline{f\left(x_{1}\right)+j_{1}}$ and $\overline{f(u)}\left(\overline{g\left(y_{1}\right)}=\overline{g\left(y_{1}\right)+j_{1}^{\prime}}\right.$ and $\overline{g(u)})$ are units in $\frac{f(A)+J}{J}\left(\frac{g(A)+J^{\prime}}{J^{\prime}}\right)$. Also, $\overline{f\left(x_{2}\right)}=\overline{f\left(x_{2}\right)+j_{2}}$ and $\overline{f(e)}($ $\overline{g\left(y_{2}\right)}=\overline{g\left(y_{2}\right)+j_{2}^{\prime}}$ and $\left.\overline{g(e)}\right)$ are idempotents in $\frac{f(A)+J}{J}\left(\frac{g(A)+J^{\prime}}{J^{\prime}}\right)$. So we have

$$
\begin{gathered}
\overline{f(a)}=\overline{f(u)}+\overline{f(e)}=\overline{f\left(x_{1}\right)}+\overline{f\left(x_{2}\right)}, \text { and } \\
\overline{g(a)}=\overline{g(u)}+\overline{g(e)}=\overline{g\left(y_{1}\right)}+\overline{g\left(y_{2}\right)} .
\end{gathered}
$$

Since $\frac{A}{I_{\circ}}$ is uniquely clean, then by Lemma $2.1, \frac{f(A)+J}{J}$ and $\frac{g(A)+J^{\prime}}{J^{\prime}}$ are uniquely clean, so

$$
\begin{gathered}
\overline{f(u)}=\overline{f\left(x_{1}\right)} \text { and } \overline{f(e)}=\overline{f\left(x_{2}\right)} \text {, and } \\
\overline{g(u)}=\overline{g\left(y_{1}\right)} \text { and } \overline{g(e)}=\overline{g\left(y_{2}\right)} \text {. }
\end{gathered}
$$

Then there exist $\left(j_{3}, j_{3}^{\prime}\right) \in J \times J^{\prime}$ and $\left(j_{4}, j_{4}^{\prime}\right) \in J \times J^{\prime}$ such that

$$
\begin{gathered}
f\left(x_{1}\right)=f(u)+j_{3} \text { and } g\left(y_{1}\right)=g(u)+j_{3}^{\prime}, \text { and } \\
f\left(x_{2}\right)=f(e)+j_{4} \text { and } g\left(y_{2}\right)=g(e)+j_{4}^{\prime} .
\end{gathered}
$$

Now,

$$
\begin{aligned}
\left(f(a)+j, g(a)+j^{\prime}\right) & =\left(f\left(x_{1}\right)+j_{1}+f\left(x_{2}\right)+j_{2}, g\left(y_{1}\right)+j_{1}^{\prime}+g\left(y_{2}\right)+j_{2}^{\prime}\right) \\
& =\left(f(u)+j_{3}+j_{1}+f(e)+j_{4}+j_{2}, g(u)+j_{3}^{\prime}+j_{1}^{\prime}+g(e)+j_{4}^{\prime}+j_{2}^{\prime}\right) \\
& =\left(f(u)+j_{3}+j_{1}, g(u)+j_{3}^{\prime}+j_{1}^{\prime}\right)+\left(f(e)+j_{4}+j_{2}, g(e)+j_{4}^{\prime}+j_{2}^{\prime}\right)
\end{aligned}
$$

Clearly, $\left(f(e)+j_{4}+j_{2}, g(e)+j_{4}^{\prime}+j_{2}^{\prime}\right)=\left(f\left(x_{2}\right)+j_{2}, g\left(y_{2}\right)+j_{2}^{\prime}\right)$ is an idempotent in $A \bowtie^{f, g}\left(J, J^{\prime}\right)$. On the other hand, since $u, f(u)+j_{3}+j_{1}$, and $g(u)+j_{3}^{\prime}+j_{1}^{\prime}$ are units in $A, f(A)+J$, and $g(A)+J^{\prime}$ respectively, then by Lemma $2.2,\left(f(u)+j_{3}+\right.$ $\left.j_{1}, g(u)+j_{3}^{\prime}+j_{1}^{\prime}\right)$ is a unit in $A \bowtie^{f, g}\left(J, J^{\prime}\right)$. Therefore, $A \bowtie^{f, g}\left(J, J^{\prime}\right)$ is clean.

Recall that if $I$ is an ideal of a ring $R$ such that $I \subseteq N i l(R)$, then $R$ is clean (nil-clean) if and only if $\frac{R}{I}$ is clean (nil-clean) [14]. Using the notation of Lemma 2.1, the following result establishes another necessary and sufficient conditions under which the bi-amalgamation will be clean.

Theorem 2.4. (1) Suppose that $I_{\circ} \subseteq \operatorname{Nil}(A)$. If $A \bowtie^{f, g}\left(J, J^{\prime}\right)$ is clean, then $A$ is clean.

(2) Suppose that $J \subseteq N i l(B)$ and $J^{\prime} \subseteq N i l(C)$. If $A$ is clean, then $A \bowtie^{f, g}\left(J, J^{\prime}\right)$ is clean.

Proof. (1) If $A \bowtie^{f, g}\left(J, J^{\prime}\right)$ is clean, then $\frac{A}{I_{\circ}} \cong \frac{A \bowtie^{f, g}\left(J, J^{\prime}\right)}{J \times J^{\prime}}$ is clean but $I_{\circ} \subseteq N i l(A)$, so $A$ is clean.

(2) If $J \subseteq N i l(B)$ and $J^{\prime} \subseteq N i l(C)$, then $J \times J^{\prime} \subseteq N i l\left(A \bowtie^{f, g}\left(J, J^{\prime}\right)\right)$. Since $A$ is clean, then so is $\frac{A}{I_{\circ}}$ but $\frac{A}{I_{\circ}} \cong \frac{A \bowtie^{f, g}\left(J, J^{\prime}\right)}{J \times J^{\prime}}$, hence $\frac{A \bowtie^{f, g}\left(J, J^{\prime}\right)}{J \times J^{\prime}}$ is clean but since $J \times J^{\prime} \subseteq \operatorname{Nil}\left(A \bowtie^{f, g}\left(J, J^{\prime}\right)\right)$, we have $A \bowtie^{f, g}\left(J, J^{\prime}\right)$ is clean.

The following lemma is used frequently in this article.

Lemma 2.5. Let $A$ be a ring, $S$ be a subring of $A$, and $I$ be an ideal of $A$. If $s \in \operatorname{Id}(S)$ and $I \subseteq I d(A)$, then $s+i \in \operatorname{Id}(A)$ for all $i \in I$. 
Proof. Since $I \subseteq I d(A)$, then $2 i=0$ and $i^{2}=i$ for all $i \in I$. So if $s \in I d(S)$ and $i \in I$, then $s^{2}=s, 2 i=0$, and $i^{2}=i$. Thus

$$
(s+i)^{2}=s^{2}+2 i s+i^{2}=s+i .
$$

The following proposition establishes (idempotent) conditions under which the bi-amalgamation will be clean.

Proposition 2.6. Suppose that $J \subseteq I d(B)$ and $J^{\prime} \subseteq I d(C)$. If $A$ is clean, then $A \bowtie^{f, g}$ $\left(J, J^{\prime}\right)$ is clean.

Proof. Let $a \in A$ and $\left(j, j^{\prime}\right) \in J \times J^{\prime}$. Then $a=u+e$ where $u \in U(A)$ and $e \in \operatorname{Id}(A)$ (since $A$ is clean). Now, since $u$ is a unit in $A$, we have $(f(u), g(u))$ is a unit in $A \bowtie^{f, g}\left(J, J^{\prime}\right)$. Also, since $e$ is an idempotent of $A$, then $f(e), g(e)$ are idempotents in $B$ and $C$ resspectively, and since $J \subseteq I d(B)$ and $J^{\prime} \subseteq I d(C)$, then by Lemma 2.5, $\left(f(e)+j, g(e)+j^{\prime}\right)$ is an idempotent in $A \bowtie^{f, g}\left(J, J^{\prime}\right)$. So, we have $\left(f(a)+j, g(a)+j^{\prime}\right)=(f(u), g(u))+\left(f(e)+j, g(e)+j^{\prime}\right)$ is a sum of a unit and an idempotent in $A \bowtie^{f, g}\left(J, J^{\prime}\right)$. Therefore, $A \bowtie^{f, g}\left(J, J^{\prime}\right)$ is clean.

Next, we appeal to the previous results to construct new examples of clean rings. Let $A$ be a ring and $M$ an $A$-module. The trivial ring extension of $A$ by $M$ is the commutative ring $A \ltimes M=A \times M$ with component-wise addition and multiplication given by $(a, m)\left(a^{\prime}, m^{\prime}\right)=\left(a a^{\prime}, a m^{\prime}+a^{\prime} m\right)$. [1].

Example 2.7. Let $A=\mathbb{Z}_{3}, B=C=A \ltimes A$, and $J=J^{\prime}=0 \ltimes \mathbb{Z}_{3}$. Consider the canonical embedding $i_{A}: A \hookrightarrow A \ltimes A(a \mapsto(a, 0))$. Since $A$ is clean and $0 \ltimes \mathbb{Z}_{3}=N i l\left(\mathbb{Z}_{3} \ltimes \mathbb{Z}_{3}\right)$, then by Theorem $2.4(2), A \bowtie^{\iota_{A}, \iota_{A}}\left(J, J^{\prime}\right)$ is clean.

The following theorem investigates a necessary and sufficient conditions for the biamalgamation to be Boolean.

Theorem 2.8. $A \bowtie^{f, g}\left(J, J^{\prime}\right)$ is Boolean if and only if $f(A), g(A)$ are Boolean, $J \subseteq \operatorname{Id}(B)$, and $J^{\prime} \subseteq I d(C)$.

Proof. Assume $A \bowtie^{f, g}\left(J, J^{\prime}\right)$ is Boolean, then $(f(a), g(a))^{2}=(f(a), g(a))$ for all $a \in A$, so $(f(a))^{2}=f(a)$ and $(g(a))^{2}=g(a)$ for all $a \in A$, it follows that $f(A)$ and $g(A)$ are Boolean. Next, let $\left(j, j^{\prime}\right) \in\left(J, J^{\prime}\right)$. Then $(j, 0)^{2}=(j, 0)$ and $\left(0, j^{\prime}\right)^{2}=\left(0, j^{\prime}\right)$, so $j^{2}=j$ and $j^{\prime 2}=j^{\prime}$. Hence, $J \subseteq \operatorname{Id}(B)$ and $J^{\prime} \subseteq \operatorname{Id}(C)$. Conversely, let $a \in A$ and $\left(j, j^{\prime}\right) \in J \times J^{\prime}$. Since $f(a), g(a)$ are idempotents in $f(A)$ and $g(A)$, respectively, $J \subseteq \operatorname{Id}(B)$ and $J^{\prime} \subseteq \operatorname{Id}(C)$, then by Lemma 2.5, $f(a)+j$ and $g(a)+j^{\prime}$ are idempotents in $B$ and $C$, respectively. Thus $\left(f(a)+j, g(a)+j^{\prime}\right)$ is idempotent in $A \bowtie^{f, g}\left(J, J^{\prime}\right)$. Therefore, $A \bowtie^{f, g}\left(J, J^{\prime}\right)$ is Boolean.

The following is a special case of Theorem 2.8 (when $f$ or $g$ is injective).

Corollary 2.9. Suppose that $f$ or $g$ is injective. Then $A \bowtie^{f, g}\left(J, J^{\prime}\right)$ is Boolean if and only if $A$ is Boolean, $J \subseteq I d(B)$, and $J^{\prime} \subseteq \operatorname{Id}(C)$.

Proof. Suppose $A \bowtie^{f, g}\left(J, J^{\prime}\right)$ is Boolean, then by Theorem 2.8, $f(A), g(A)$ are Boolean, $J \subseteq I d(B)$, and $J^{\prime} \subseteq I d(C)$. If $f$ or $g$ is injective, then $A \cong f(A)$ or $A \cong g(A)$ respectively, and in both cases $A$ is Boolean. Conversely, suppose $A$ is Boolean, then $f(A)$ and $g(A)$ are Boolean, and since $J \subseteq I d(B)$, and $J^{\prime} \subseteq I d(C)$, then by Theorem 2.8, $A \bowtie^{f, g}\left(J, J^{\prime}\right)$ is Boolean.

Corollary 2.9 can be used to recover an old result by Mohamed Chhiti, Najib Mahdou and Mohammed Tamekkante in [5]

Corollary 2.10 ([5, Proposition $2.19(2)]$ ). Let $f: A \rightarrow B$ be a ring homomorphism and $J$ be an ideal B. Then $A \bowtie^{f} J$ is Boolean if and only if $A$ is Boolean and $J \subseteq \operatorname{Id}(B)$. 
The following lemma is useful in testing the transfer of the nil-clean in the bi-amalgamation.

Lemma 2.11. Let $f: A \rightarrow B$ be a ring homomorphism and let $J$ be an ideal of $B$. Then

(1) If $A$ is nil-clean and $J \subseteq N i l(B)$, then $f(A)+J$ is nil-clean.

(2) If $A$ is nil-clean and $J \subseteq I d(B), f(A)+J$ is nil-clean.

Proof. (1) Let $a \in A$ and $j \in J$. Since A is nil-clean, there exists an idempotent $e$ and a nilpotent $n$ such that $a=e+n$. So, $f(a)+j=f(e)+f(n)+j$. Clearly, $f(e) \in \operatorname{Id}(f(A)+J)$ and $f(n) \in \operatorname{Nil}(f(A)+J)$, but since $j \in J \subseteq \operatorname{Nil}(B)$, we have $f(n)+j \in N i l(f(A)+J)$. Hence, $f(a)+j=f(e)+(f(n)+j)$ is sum of an idempotent and a nilpotent in $f(A)+J$. Therefore, $f(A)+J$ is nil-clean.

(2) Let $a \in A$ and $j \in J$. Since $\mathrm{A}$ is nil-clean, there exists an idempotent $e$ and a nilpotent $n$ such that $a=e+n$. So, $f(a)+j=f(e)+f(n)+j=(f(e)+j)+f(n)$. Clearly, $f(n) \in \operatorname{Nil}(f(A)+J)$, and since $f(e)$ is idempotent in $f(A)$ and $J \subseteq \operatorname{Id}(B)$, then by Lemma 2.5, $f(e)+j$ is an idempotent in $f(A)+J$. Hence, $f(a)+j=$ $(f(e)+j)+f(n)$ is sum of an idempotent and a nilpotent in $f(A)+J$. Therefore, $f(A)+J$ is nil-clean.

Recall that a commutative ring $R$ is nil-clean if and only if for all $r \in R, r-r^{2}$ is nilpotent [13, Theorem 3]. The following theorem provides the necessary and sufficient conditions for the bi-amalgamation to inherit the nil-clean property.

Theorem 2.12. . Let $A \bowtie^{f, g}\left(J, J^{\prime}\right)$ be the bi-amalgamation of $A$. Then the following conditions are equivalent:

(1) $A \bowtie^{f, g}\left(J, J^{\prime}\right)$ is nil-clean.

(2) $f(A)+J$ and $g(A)+J^{\prime}$ are nil-clean.

Proof. $(1) \Rightarrow(2)$. Assume $A \bowtie^{f, g}\left(J, J^{\prime}\right)$ is nil-clean. Then $f(A)+J$ and $g(A)+J^{\prime}$ are nil-clean rings since by Lemma 2.1, they are homomorphic images of $A \bowtie^{f, g}\left(J, J^{\prime}\right)$ which is nil-clean.

$(2) \Rightarrow(1)$. Let $a \in A$ and $\left(j, j^{\prime}\right) \in J \times J^{\prime}$. By $(2),(f(a)+j)-(f(a)+j)^{2}$ and $\left(g(a)+j^{\prime}\right)-\left(g(a)+j^{\prime}\right)^{2}$ are nilpotents $\operatorname{in} f(A)+J$ and $g(A)+J^{\prime}$, respectively. Hence, $\left(f(a)+j, g(a)+j^{\prime}\right)-\left(f(a)+j, g(a)+j^{\prime}\right)^{2}$ is nilpotent in $A \bowtie^{f, g}\left(J, J^{\prime}\right)$. Therefore, $A \bowtie^{f, g}\left(J, J^{\prime}\right)$ is nil-clean.

The following result was proved by Chahrazade Bakkari and Mohamed Es-Said in [2] which can be recovered by using Theorem 2.12 .

Corollary 2.13 ([2, Theorem 2.1]). Let $f: A \rightarrow B$ be a ring homomorphism and let $J$ be an ideal of $B$. Then the following conditions are equivalent:

(1) $A \bowtie^{f} J$ is nil-clean.

(2) $A$ and $f(A)+J$ are nil-clean.

The following is the special case of Theorem 2.12 when $A$ is nil-clean.

Corollary 2.14. Let $A \bowtie^{f, g}\left(J, J^{\prime}\right)$ be the bi-amalgamation of $A$.

(1) Suppose that $J \subseteq N i l(B)$ and $J^{\prime} \subseteq N i l(C)$. If $A$ is nil-clean, then $A \bowtie^{f, g}\left(J, J^{\prime}\right)$ is nil-clean.

(2) Suppose that $J \subseteq I d(B)$ and $J^{\prime} \subseteq I d(C)$. If $A$ is nil-clean, then $A \bowtie^{f, g}\left(J, J^{\prime}\right)$ is nil-clean.

Proof. (1) This follows from Lemma 2.11 (1) and Theorem 2.12.

(2) This follows from Lemma 2.11 (2) and Theorem 2.12.

The following example uses Corollary 2.14 to build new examples of nil-clean rings. 
Example 2.15. Let $A=\mathbb{Z}_{6} \ltimes \mathbb{Z}_{6}$. Since $\mathbb{Z}_{6}$ is a nil-clean ring, then by 112 , Corollary 3.23 (1)], $A$ is nil-clean. Now, consider the ring homomorphism $p_{1}: A \rightarrow \mathbb{Z}_{6}((a, b) \mapsto a)$. Since $\langle 3\rangle \subset \operatorname{Id}\left(\mathbb{Z}_{6}\right)$, then by Corollary $2.14(2), A \bowtie^{p_{1}, p_{1}}(\langle 3\rangle,\langle 3\rangle)$ is nil-clean.

Proposition 2.16. Suppose that $I_{\circ} \subseteq \operatorname{Nil}(A)$. If $A \bowtie^{f, g}\left(J, J^{\prime}\right)$ is nil-clean, then $A$ is nil-clean.

Proof. Assume that $A \bowtie^{f, g}\left(J, J^{\prime}\right)$ is nil-clean, then $\frac{A}{I_{\circ}} \cong \frac{A \bowtie^{f, g}\left(J, J^{\prime}\right)}{J \times J^{\prime}}$ is nil-clean but $I_{\circ} \subseteq N i l(A)$, so $A$ is nil-clean.

The case of $f$ and $g$ are surjective maps can give a necessary and sufficient conditions for $A \bowtie^{f, g}\left(J, J^{\prime}\right)$ to be (nil-)clean.

Proposition 2.17. Consider $A \bowtie^{f, g}\left(J, J^{\prime}\right)$ with $f$ and $g$ are surjective maps. If $I_{\circ} \subseteq$ $\operatorname{Nil}(A)$, then $A \bowtie^{f, g}\left(J, J^{\prime}\right)$ is clean (nil-clean) if and only if $A$ is clean (nil-clean).

Proof. Since $I_{\circ} \subseteq N i l(A)$, then by Theorem 2.4 (1) (Proposition 2.16), $A \bowtie^{f, g}\left(J, J^{\prime}\right)$ is clean (nil-clean) implies $A$ is clean (nil-clean). Conversely, since $f$ and $g$ are surjective and $I_{\circ} \subseteq N i l(A)$, then $J \subseteq N i l(B)$ and $J^{\prime} \subseteq N i l(C)$, so by Theorem 2.4 (2) (Corollary $2.14(1)), A$ is clean (nil-clean) implies $A \bowtie^{f, g}\left(J, J^{\prime}\right)$ is clean (nil-clean).

Lastly, we use Theorem 2.12 to provide another necessary and sufficient conditions for the bi-amalgamation to inherit the nil-clean property.

Theorem 2.18. Let $A \bowtie^{f, g}\left(J, J^{\prime}\right)$ be the bi-amalgamation of $A$. Then the following conditions are equivalent:

(1) $A \bowtie^{f, g}\left(J, J^{\prime}\right)$ is nil-clean.

(2) Any proper homomorphic image of $A \bowtie^{f, g}\left(J, J^{\prime}\right)$ is nil-clean.

Proof. Since any proper homomorphic image of a nil-clean ring is nil-clean, then the implication $(1) \Rightarrow(2)$ holds. For the other implication, since $f(A)+J$ and $g(A)+J^{\prime}$ are proper homomorphic image of $A \bowtie^{f, g}\left(J, J^{\prime}\right)$, they are nil-clean, hence by Theorem 2.12, $A \bowtie^{f, g}\left(J, J^{\prime}\right)$ is nil-clean.

\section{References}

[1] D.D. Anderson and M. Winders, Idealization of a module, J. Commut. Algebra, 1 (1), 3-56, 2009.

[2] C. Bakkari and M. Es-Saidi, Nil-clean property in amalgamated algebras along an ideal, Ann Univ Ferrara 65 (1), 15-20, 2019.

[3] F. Campanini and C.A. Finocchiaro, Bi-amalgamated constructions, J. Algebra Appl. 18 (8), 1950148, 2019.

[4] M. Chhiti, M. Jarrar, S. Kabbaj and N. Mahdou, Prüfer conditions in an amalgamated duplication of a ring along an ideal, Comm. Algebra, 43 (1), 249-261, 2015.

[5] M. Chhiti, N. Mahdou and M. Tamekkante, Clean property in amalgamated algebras along an ideal, Hacet. J. Math. Stat. 44 (1), 41-49, 2015.

[6] M. D'Anna, A construction of Gorenstein rings, J. Algebra, 306, 507-519, 2006.

[7] M. D'Anna, C.A. Finocchiaro and M. Fontana, Amalgamated algebras along an ideal, in: Commutative Algebra and Applications, Proceedings of the Fifth International Fez Conference on Commutative Algebra and Applications, Fez, Morocco, 2008, W. de Gruyter Publisher, Berlin, 155-172, 2009.

[8] M. D'Anna, C.A. Finocchiaro and M. Fontana, New Algebraic Properties of an Amalgamated Algebra Along an Ideal, Comm. Algebra, 44 (5), 1836-1851, 2016.

[9] M. D'Anna, C.A. Finocchiaro and M. Fontana, Properties of chains of prime ideals in amalgamated algebras along an ideal, J. Pure Appl. Algebra, 214, 1633-1641, 2010.

[10] M. D'Anna and M. Fontana, An amalgamated duplication of a ring along an ideal: the basic properties, J. Algebra Appl. 6 (3), 443-459, 2007. 
[11] M. D'Anna and M. Fontana, The amalgamated duplication of a ring along a multiplicative canonical ideal, Ark. Mat. 45 (2), 241-252, 2007.

[12] A.J. Diesl, Nil clean rings, J. Algebra 383, 197-211, 2013.

[13] Y. Hirano, H. Tominaga and A. Yaqub, On rings in which every element is uniquely expressible as a sum of a nilpotent element and a certain potent element, Math. J. Okayama Univ. 30, 33-40, 1988.

[14] N.A. Immormino, Clean rings and clean group rings, PhD Dissertation, Bowling Green State University, 2013.

[15] S. Kabbaj, K. Louartiti, and M. Tamekkante, Bi-amalgamated algebras along ideals, J. Commut. Algebra, 9 (1), 65-87, 2017.

[16] S. Kabbaj, N. Mahdou and M.A.S. Moutui, Bi-amalgamations subject to the arithmetical property, J. Algebra Appl. 16 (2), 1750030, 2017.

[17] W. Nicholson, Lifting idempotents and exchange rings, Trans. Am. Math. Soc. 229, 269-278, 1977. 